\title{
On a characterization of distributive rings via saturations and its applications to Armendariz and Gaussian rings
}

\author{
Ryszard Mazurek and Michał Ziembowski
}

\begin{abstract}
In this paper we apply Ferrero-Sant'Ana's characterization of right distributive rings via saturations to prove that all right distributive rings are Armendariz relative to any unique product monoid. As an immediate consequence we obtain that all right distributive rings are Armendariz. We apply this result to give a new proof of the well-known fact that all right duo right distributive rings are right Gaussian. We also show that for any nontrivial unique product monoid $S$, the class of Armendariz rings relative to $S$ is contained in the class of Armendariz rings, and we present an example of a unique product monoid $S$ for which this containment is strict.
\end{abstract}

\section{Introduction}

Throughout this paper, $R$ denotes an associative ring with unity. Recall that a ring $R$ is said to be right distributive if the lattice of right ideals of $R$ is distributive, i.e., $(A+B) \cap C=A \cap C+B \cap C$ for any right ideals $A, B$ and $C$ of $R$. Left distributive rings are defined similarly. A $\operatorname{ring} R$ is called an Armendariz ring if whenever the product of two polynomials over $R$ is zero, then the products of their coefficients are all zero, that is, for any $f=\sum_{i=0}^{m} a_{i} x^{i}$ and $g=\sum_{j=0}^{n} b_{j} x^{j} \in$ $R[x]$, if $f g=0$, then $a_{i} b_{j}=0$ for all $i$ and $j$. These rings were introduced by M. B. Rege and S. Chhawchharia in [20], and the name for them was chosen to honor E. P. Armendariz, who noted in [2] that all reduced rings satisfy this condition. Armendariz rings, as well as their numerous generalizations (see [15]), have recently been objects of intensive investigation.

The aim of this paper is to examine relationships between the classes of right distributive rings and Armendariz rings. The main motivation for the study comes from the theory of commutative rings. According to L. Fuchs, [8], commutative

Mathematics Subject Classification (2010): Primary 16S36, 16U99; Secondary 16U20, 20 M25.

Keywords: Right distributive ring, Armendariz ring, Armendariz ring relative to a monoid, right Gaussian ring, saturation of a right ideal, unique product monoid. 
distributive rings are called arithmetical rings. It is well known that a commutative domain is arithmetical if and only if it is Prüfer if and only if it is Gaussian (see, e.g., [9]), where a commutative ring $R$ is said to be Gaussian if

$$
c(f g)=c(f) c(g) \text { for any } f, g \in R[x]
$$

with $c(h)$ denoting the ideal of $R$ generated by the coefficients of a polynomial $h \in R[x]$. Thus in the commutative case, both arithmetical rings and Gaussian rings generalize the important notion of a Prüfer domain to rings with zero divisors. When passing from commutative domains to all commutative rings, one implication between the arithmetical and the Gaussian conditions remains valid; namely if a ring is arithmetical then it is Gaussian (see Section 4 of [9]). As proved by D. D. Anderson and V. Camillo in Theorem 1 in [1], a commutative ring is Gaussian if and only if all its homomorphic images are Armendariz rings. Therefore, since obviously homomorphic images of arithmetical rings are arithmetical, the aforementioned result,

$$
R \text { is an arithmetical ring } \Rightarrow R \text { is a Gaussian ring, }
$$

can be seen as an immediate consequence of the implication

$$
R \text { is an arithmetical ring } \Rightarrow R \text { is an Armendariz ring, }
$$

which is explicitly outlined, for example, as Corollary 9.56 in C. Faith's book [6].

In this paper we extend the result (1.3) to noncommutative rings. Namely we show that for any ring $R$,

$$
R \text { is a right distributive ring } \Rightarrow R \text { is an Armendariz ring. }
$$

In fact we will prove the much stronger result,

$$
R \text { is a right distributive ring }
$$

$\Rightarrow R$ is an Armendariz ring relative to any u.p. monoid $S$,

where according to [13], a ring $R$ is said to be Armendariz relative to a monoid $S$, or $S$-Armendariz for short, if for any elements $\alpha=\sum_{s \in S} a_{s} s$ and $\beta=\sum_{t \in S} b_{t} t$ of the monoid ring $R[S]$ (where $s, t \in S$ and $a_{s}, b_{t} \in R$ ), $\alpha \beta=0$ implies $a_{s} b_{t}=0$ for all $s, t \in S$ (the definition of a u.p. monoid is recalled at the beginning of Section 4). Since the additive monoid $S=\mathbb{N} \cup\{0\}$ of nonnegative integers is a u.p. monoid and the monoid ring $R[S]$ is isomorphic to the polynomial ring $R[x]$, it follows that (1.4) is a consequence of (1.5). To show that the result (1.5) is indeed stronger than (1.4), we will construct an example of an Armendariz ring that is not $S$-Armendariz for some u.p. monoid $S$.

One advantage of proving the implication (1.4) is that every property established for Armendariz rings is automatically inherited by right distributive rings. For example, the property that nilpotent elements of a right distributive ring $R$ form a (nonunital) subring of $R$, proved in Corollary 2 in [16], can now be seen as an 
immediate consequence of the same property for nilpotent elements of Armendariz rings, proved in Proposition 4.5 in [15].

Another benefit of proving the result (1.4) is that it enlarges the stock of examples of Armendariz rings to include all known examples of right distributive rings. Since the structure of right distributive rings is quite subtle, these examples can be helpful in developing the theory of Armendariz rings.

Yet another benefit from proving (1.4) is a new proof of the well-known fact that right duo right distributive rings are right Gaussian, which will be presented in Section 4. This fact extends (1.2) to the noncommutative setting. Right Gaussian rings were introduced in [17] by replacing each ideal $c(h)$ in the condition (1.1) with the right ideal $c_{r}(h)$ generated by the coefficients of a polynomial $h \in R[x]$.

The paper is organized as follows. Section 2 is devoted to so-called saturations, which are some special right ideals of a ring, used by M. Ferrero and A. Sant'Ana in [7] to characterize right distributive rings in the spirit of a well-known characterization by Jensen of commutative distributive rings. In Section 2 we point out some links between the result of Ferrero-Sant'Ana and that of Jensen, and collect properties of saturations needed in later parts of the paper. In Section 3 we give an alternative proof of the Ferrero-Sant'Ana result, which is then used in Section 4 to prove that all right distributive rings are Armendariz relative to any u.p. monoid. In Section 5 we show that for any nontrivial u.p. monoid $S$, the class of Armendariz rings relative to $S$ is contained in the class of Armendariz rings, and we present an example of a u.p. monoid $S$ for which this containment is strict.

\section{Saturations of right ideals}

In 1964, C. U. Jensen proved the following characterization of arithmetical rings (i.e., commutative distributive rings).

Theorem 2.1. (Lemma 1 in [10]) $A$ commutative ring $R$ is arithmetical if and only if for any maximal ideal $M$ of $R$, the ideals of the ring of fractions of $R$ with respect to $D=R \backslash M$ are totally ordered by set inclusion.

Rings of fractions, as shown by the result of Jensen, are very useful tools in studying commutative distributive rings. In the noncommutative setting the situation is different, as for a right distributive ring $R$ the ring of fractions with respect to the complement $D=R \backslash M$ of a maximal right ideal $M$ of $R$ may not exist (see Section 7 of [19]). In [7] Ferrero and Sant'Ana have overcome the problem by adjoining to any right ideal $I$ of $R$ its $D$-saturation

$$
I D^{-1}=\{r \in R: r d \in I \text { for some } d \in D\}, \quad \text { where } D=R \backslash M,
$$

and considering the lattice of $D$-saturations of right ideals of $R$ instead of the lattice of right ideals of the possibly nonexistent ring of fractions with respect to $D$. They proved in Corollary 3.6 in [7] that a ring $R$ is right distributive if and only if for any maximal right ideal $M$ of $R$ the $D$-saturations of right ideals of $R$ are totally ordered by set inclusion. An alternative proof of this result will be 
given in Section 3. In the present section we explain why the result of Ferrero and Sant'Ana is for noncommutative rings an adequate analogue of the result of Jensen. Furthermore, since saturations play a crucial role in Section 4 in our proof that all right distributive rings are $S$-Armendariz for any u.p. monoid $S$, we establish the properties of saturations that will be needed in the proof.

Let $R$ be a ring and let $D$ be a multiplicative subset of $R$ (i.e., $D \cdot D \subseteq D$, $1 \in D$, and $0 \notin D)$. Then a ring $R_{D}$ is called a right ring of fractions of $R$ with respect to $D$ if there exists a ring homomorphism $\varphi: R \rightarrow R_{D}$ such that

(a) For any $d \in D, \varphi(d)$ is an invertible element of $R_{D}$.

(b) Every element of $R_{D}$ has the form $\varphi(r) \varphi(d)^{-1}$ for some $r \in R$ and $d \in D$.

(c) $\operatorname{ker} \varphi=\{r \in R: r d=0$ for some $d \in D\}$.

It is well known (e.g. see Theorem 10.6 in [12]) that the ring $R$ has a right ring of fractions with respect to $D$ if and only if the following conditions are satisfied:

(1) For any $r \in R$ and $d \in D, r D \cap d R \neq \emptyset$.

(2) For any $r \in R$, if $d^{\prime} r=0$ for some $d^{\prime} \in D$, then $r d=0$ for some $d \in D$.

If a set $D \subseteq R$ satisfies property (1) above, then $D$ is said to be right Ore, whereas a set $D \subseteq R$ satisfying property (2) is said to be right reversible.

A right ideal $P$ of a ring $R$ such that the complement $D=R \backslash P$ is a multiplicative subset of $R$ is said to be completely prime. In other words, a right ideal $P$ of a ring $R$ is completely prime if $P \neq R$ and for any $a, b \in R, a b \in P$ implies $a \in P$ or $b \in P$.

It is well known that any maximal right ideal $M$ of a right distributive ring $R$ is a two-sided ideal of $R$ (see Corollary 4 of Proposition 1.1 in [21]) and thus $M$ is completely prime. Hence the complement $D=R \backslash M$ is a multiplicative subset of $R$. In fact we have the following result.

Proposition 2.2. (Theorem 7.7 (1) in [23]) If $R$ is a right distributive ring and $M$ is a maximal right ideal of $R$, then $D=R \backslash M$ is a right Ore, multiplicative subset of $R$.

Unfortunately, as the example constructed by G. Puninski in Section 7 of [19] shows, the complement $D=R \backslash M$ of a maximal right ideal $M$ of a right distributive ring $R$ need not be right reversible, so right rings of fractions with respect to $D=R \backslash M$ may not exist. Therefore, it is impossible to extend Jensen's Theorem 2.1 to right distributive rings simply by using right rings of fractions. To overcome this difficulty we consider some special right ideals of a right distributive ring $R$ that exist independently of whether or not $D=R \backslash M$ is right reversible and, in the case where $D$ is right reversible, furthermore constitute a lattice isomorphic to the lattice of right ideals of the right ring of fractions $R_{D}$.

Let $R$ be any ring and let $D$ be any nonempty subset of $R$. Following [7], for a right ideal $I$ of $R$ we define the saturation of $I$ with respect to $D$, or $D$-saturation of $I$ for short, to be the set

$$
I D^{-1}=\{r \in R: r d \in I \text { for some } d \in D\}
$$


(as is pointed out in [7], this notion has also been used by G. Törner and J. Zima in [22] to study right distributive domains). Assume that $D$ is a multiplicative, right Ore subset of $R$. Then as proved in Proposition 2.4 below, for any right ideal $I$ of $R$ the $D$-saturation $I D^{-1}$ of $I$ is a right ideal of $R$. Let

$$
\mathcal{L}=\left\{I D^{-1}: I \text { is a right ideal of } R\right\},
$$

i.e., $\mathcal{L}$ is the set of all $D$-saturations of right ideals of $R$. Then ordered by inclusion, $\mathcal{L}$ has a lattice structure, where for $J, K \in \mathcal{L}$ the meet and join are defined by

$$
J \wedge K=J \cap K \quad \text { and } \quad J \vee K=(J+K) D^{-1} .
$$

Assume for a moment that furthermore $D$ is right reversible. Then the $\operatorname{ring} R_{D}$ of right fractions of $R$ with respect to $D$ exists. Let $\varphi$ be the natural homomorphism from $R$ to $R_{D}$. For any $J \in \mathcal{L}$ we set $\Psi(J)=\varphi(J) \cdot R_{D}$, the right ideal generated by $\varphi(J)$ in $R_{D}$. It is easy to verify that $\Psi$ is a lattice isomorphism from the lattice $\mathcal{L}$ of $D$-saturations of right ideals of $R$ onto the lattice of right ideals of $R_{D}$. Hence the following result of Ferrero and Sant'Ana is an adequate generalization of Jensen's Theorem 2.1 to noncommutative rings.

Theorem 2.3. (Corollary 3.6 in [7]) $A$ ring $R$ is right distributive if and only if for any maximal right ideal $M$ of $R$, the saturations of right ideals of $R$ with respect to $D=R \backslash M$ are totally ordered by set inclusion.

An alternative proof of the Ferrero-Sant'Ana result will be given in Section 3. Below we prove those properties of saturations that will be needed in Section 4 in proving the main result of this paper, namely that all right distributive rings are Armendariz relative to any u.p. monoid.

Proposition 2.4. Let $R$ be a ring and let $D$ be a multiplicative, right Ore subset of $R$. Then:

(a) For any right ideal $I$ of $R$ and any $n \in \mathbb{N}$, if $a_{1}, a_{2}, \ldots, a_{n} \in I D^{-1}$, then there exists $d \in D$ such that $a_{1} d, a_{2} d, \ldots, a_{n} d \in I$.

(b) For any right ideal $I$ of $R, I D^{-1}$ is a right ideal of $R$ and $I \subseteq I D^{-1}$.

(c) For any right ideals $I, J$ of $R$,

$$
J \subseteq I D^{-1} \text { if and only if } J D^{-1} \subseteq I D^{-1} .
$$

(d) For any right ideal $I$ of $R,\left(I D^{-1}\right) D^{-1}=I D^{-1}$.

(e) For any right ideals $I, J$ of $R$, if $I D^{-1} \subseteq J D^{-1}$, then $(a I) D^{-1} \subseteq(a J) D^{-1}$ for any $a \in R$.

Proof. (a) The case where $n=1$ is obvious. Assume $n \geq 2, a_{1}, a_{2}, \ldots, a_{n} \in I D^{-1}$ and the property (a) is true for any $n-1$ elements of $I D^{-1}$. Then by the inductive hypothesis there exists $d^{\prime} \in D$ such that $a_{1} d^{\prime}, a_{2} d^{\prime}, \ldots, a_{n-1} d^{\prime} \in I$. Since $a_{n} \in I D^{-1}$, for some $d^{\prime \prime} \in D_{\sim}$ we have $a_{n} d^{\prime \prime} \in I_{2}$ and since $D$ is right Ore, there exist $\widetilde{d} \in D$ and $r \in R$ with $d^{\prime} \widetilde{d}=d^{\prime \prime} r$. Set $d=d^{\prime} \widetilde{d}$. Then $d \in D, a_{i} d=\left(a_{i} d^{\prime}\right) \widetilde{d} \in I$ for any $i \in\{1,2, \ldots, n-1\}$, and $a_{n} d=\left(a_{n} d^{\prime \prime}\right) r \in I$. 
(b) If $a \in I$, then $a \cdot 1 \in I$ and $1 \in D$, and thus $a \in I D^{-1}$. Hence $I \subseteq I D^{-1}$. In particular, $I D^{-1} \neq \emptyset$.

Let $a_{1}, a_{2} \in I D^{-1}$. Then by (a) there exists $d \in D$ such that $a_{1} d, a_{2} d \in I$, and since $\left(a_{1}+a_{2}\right) d=a_{1} d+a_{2} d \in I$, it follows that $a_{1}+a_{2} \in I D^{-1}$.

Let $a \in I D^{-1}$ and $r \in R$. Then $a d \in I$ for some $d \in D$, and since $D$ is right Ore, $r d_{1}=d r_{1}$ for some $d_{1} \in D$ and $r_{1} \in R$. Since $a r d_{1}=a d r_{1} \in I r_{1} \subseteq I$ and $d_{1} \in D$, ar $\in I D^{-1}$.

(c) Assume $J \subseteq I D^{-1}$, and let $a \in J D^{-1}$. Then $a d \in J$ for some $d \in D$, and thus $a d \in I D^{-1}$. Hence $a d d_{1} \in I$ for some $d_{1} \in D$. Since $d d_{1} \in D, a \in I D^{-1}$ and thus $J D^{-1} \subseteq I D^{-1}$.

Assume $J D^{-1} \subseteq I D^{-1}$. Then (b) implies that $J \subseteq I D^{-1}$.

(d) If $a \in\left(I D^{-1}\right) D^{-1}$, then $a d_{1} \in I D^{-1}$ and $a d_{1} d_{2} \in I$ for some $d_{1}, d_{2} \in D$. Since $d_{1} d_{2} \in D$, it follows that $a \in I D^{-1}$ and thus $\left(I D^{-1}\right) D^{-1} \subseteq I D^{-1}$. The opposite inclusion is a consequence of (b).

(e) Assume that $I D^{-1} \subseteq J D^{-1}$, and let $x \in(a I) D^{-1}$. Then $x d_{1}=a i$ for some $d_{1} \in D$ and $i \in I$. Since $I \subseteq I D^{-1} \subseteq J D^{-1}$, there exists $d_{2} \in D$ such that $i d_{2} \in J$. Now for $d=d_{1} d_{2} \in D$ we have $x d=\left(x d_{1}\right) d_{2}=a\left(i d_{2}\right) \in a J$, and thus $x \in(a J) D^{-1}$, which shows that $(a I) D^{-1} \subseteq(a J) D^{-1}$.

We will consider mainly saturations with respect to complements of completely prime right ideals. Such saturations have the following property.

Corollary 2.5. Let $P$ be a completely prime right ideal of a ring $R$ such that $D=R \backslash P$ is a right Ore subset of $R$. Then, for any element $a \in R$,

(a) if $I$ is a right ideal of $R$ and $I D^{-1} \varsubsetneqq(a R) D^{-1}$, then $I D^{-1} \subseteq(a P) D^{-1}$;

(b) if $a \in(a P) D^{-1}$, then $a \in 0 D^{-1}$.

Proof. (a) Let $x \in I D^{-1}$. Since $I D^{-1} \subseteq(a R) D^{-1}$, there exist $d \in D$ and $r \in R$ such that $x d=a r$. If $r \in D$, then since $a r=x d \in I D^{-1}$ by Proposition $2.4(\mathrm{~b})$, it follows from Proposition $2.4(\mathrm{~d})$ that $a \in\left(I D^{-1}\right) D^{-1}=I D^{-1}$, so $a R \subseteq I D^{-1}$ by Proposition 2.4 (b), and applying Proposition 2.4 (c) we come to the contradiction $(a R) D^{-1} \subseteq I D^{-1}$. Hence $r \notin D$, so $r \in P$ and the equality $x d=a r$ implies $x \in(a P) D^{-1}$, as desired.

(b) Since $a \in(a P) D^{-1}$, $a d=a p$ for some $d \in D$ and $p \in P$. Hence we have $a(d-p)=0$ with $d-p \in D$, and thus $a \in 0 D^{-1}$.

\section{A characterization of right distributive rings via satura- tions}

The main result of this paper is Theorem 4.1, which says that every right distributive ring is Armendariz relative to any u.p. monoid. As already mentioned, the proof of the result applies the Ferrero-Sant'Ana characterization of right distributive rings via saturations established in Corollary 3.6 in [7]. For the sake of completeness, we give below a self-contained proof of the characterization, which is 
different than the proof given by Ferrero and Sant'Ana in [7]. (Another reason for including the proof is that our definition of a saturation is more general than that in [7] and, furthermore, condition (iii) below differs slightly from the corresponding condition (iii) in Corollary 3.6 of [7].)

Theorem 3.1. For any ring $R$ the following conditions are equivalent:

(i) $R$ is right distributive.

(ii) For any maximal right ideal $M$ of $R$, the saturations of right ideals of $R$ with respect to $D=R \backslash M$ are totally ordered by set inclusion.

(iii) For any maximal right ideal $M$ of $R$ and any elements $a, b \in R$,

$$
(a R) D^{-1} \subseteq(b R) D^{-1} \text { or }(b R) D^{-1} \subseteq(a R) D^{-1},
$$

where $D=R \backslash M$.

Proof. (i) $\Rightarrow$ (ii) Assume $R$ is right distributive and $M$ is a maximal right ideal of $R$. Then by Proposition $2.2, D=R \backslash M$ is a right Ore multiplicative subset of $R$. Suppose there exist right ideals $I, J$ of $R$ such that $I D^{-1} \not J D^{-1}$ and $J D^{-1} \nsubseteq I D^{-1}$, and choose $a \in I D^{-1} \backslash J D^{-1}$ and $b \in J D^{-1} \backslash I D^{-1}$. Since $R$ is right distributive,

$$
a R=a R \cap(b R+(a-b) R)=a R \cap b R+a R \cap(a-b) R .
$$

Hence $a=c+a h$, where $c \in a R \cap b R, h \in R$, and $a h=(a-b) r$ for some $r \in R$. If $h \in M$, then $1-h \in D$, and since by Proposition 2.4 (b) we have $a(1-h)=c \in b R \subseteq J D^{-1}$, it follows from Proposition $2.4(\mathrm{~d})$ that $a \in J D^{-1}$, a contradiction. If $r \in D$, then $b r=a(r-h) \in a R \subseteq I D^{-1}$ implies that $b \in I D^{-1}$, a contradiction. We are left with the case where $h \in D$ and $r \in M$. Then $r-h \in D$, and since $a(r-h)=b r \in b R \subseteq J D^{-1}$, we again obtain a contradiction $a \in J D^{-1}$. Hence for any right ideals $I, J$ of $R$ we have $I D^{-1} \subseteq J D^{-1}$ or $J D^{-1} \subseteq I D^{-1}$, which proves (ii).

(ii) $\Rightarrow$ (iii) This is obvious.

(iii) $\Rightarrow$ (i) Assume (iii). We have to show that $(A+B) \cap C=A \cap C+B \cap C$ for any right ideals $A, B$ and $C$ of $R$. Obviously, $A \cap C+B \cap C \subseteq(A+B) \cap C$. To show the opposite inclusion, let $r \in(A+B) \cap C$. Then $r \in C$ and $r=a+b$ for some $a \in A$ and $b \in B$. It is clear that the set

$$
I=\{x+y: x, y \in R, r x \in A, r y \in B\}
$$

is a right ideal of $R$. Suppose $I \neq R$. Then $I \subseteq M$ for a maximal right ideal $M$ of $R$. Set $D=R \backslash M$. Then by (iii) we have $(a R) D^{-1} \subseteq(b R) D^{-1}$ or $(b R) D^{-1} \subseteq$ $(a R) D^{-1}$. If $(a R) D^{-1} \subseteq(b R) D^{-1}$, then $a \in(a R) D^{-1} \subseteq(b R) D^{-1}$, and thus $a d \in b R \subseteq B$ for some $d \in D$. However, then $r d=(a+b) d=a d+b d \in B$, so $d=0+d \in I \subseteq M$, a contradiction. Similarly we get a contradiction if $(b R) D^{-1} \subseteq(a R) D^{-1}$. Hence $I=R$, and thus there exist $x, y \in R$ with $x+y=1$, $r x \in A$ and $r y \in B$. Therefore, $r=r(x+y)=r x+r y \in A \cap C+B \cap C$, as desired. 


\section{Right distributive rings are Armendariz relative to u.p. monoids}

In this section we will prove that every right or left distributive ring is Armendariz relative to any u.p. monoid. Recall that a monoid $S$ is said to be a unique product monoid (a u.p. monoid for short) if for any two nonempty finite subsets $X, Y \subseteq S$ there exist $x_{0} \in X$ and $y_{0} \in Y$ such that $x_{0} y_{0} \neq x y$ for every $(x, y) \in X \times$ $Y \backslash\left\{\left(x_{0}, y_{0}\right)\right\}$; the element $x_{0} y_{0}$ is called a u.p. element of $X Y=\{s t: s \in S, t \in Y\}$. The class of u.p. monoids is quite large and important (e.g. it includes totally ordered monoids, submonoids of a free group, torsion-free nilpotent groups, and a.n.u.p.-monoids; see [14] for the definition of the last notion), and u.p. monoids have been considered in many papers (e.g. see [5], [14], [15], and [18]).

Theorem 4.1. Let $R$ be a right or left distributive ring and let $S$ be a u.p. monoid. Then $R$ is Armendariz relative to $S$.

The proof of the theorem will be based on the following observation.

Lemma 4.2. Let $R$ be a ring and let $P$ be a completely prime right ideal of $R$ such that $D=R \backslash P$ is a right Ore subset of $R$ and the saturations of right ideals of $R$ with respect to $D$ are totally ordered by set inclusion. Let $S$ be a u.p. monoid and let $\alpha=\sum_{s \in S} a_{s} s, \beta=\sum_{t \in S} b_{t} t \in R[S]$ be such that $\alpha \beta=0$. Then there exists $d \in D$ such that $a_{s} b_{t} d=0$ for any $s, t \in S$.

Proof. In the proof we adapt some ideas of the proof of Proposition 6.1 in [15]. For any $\gamma=\sum_{s \in S} c_{s} s \in R[S], c_{s}$ is called the $s$-coefficient of $\gamma$, and $\operatorname{supp}(\gamma)$ denotes the support of $\gamma$, i.e., $\operatorname{supp}(\gamma)=\left\{s \in S: c_{s} \neq 0\right\}$.

If $\alpha=0$ or $\beta=0$, it suffices to take $d=1$. Thus we can assume that $\alpha \neq 0$ and $\beta \neq 0$. By Proposition 2.4 (a), to prove the result, it suffices to show that $a_{s} b_{t} \in 0 D^{-1}$ for any $s, t \in S$.

Since the saturations of right ideals of $R$ with respect to $D$ are totally ordered, there exist $s_{0} \in \operatorname{supp}(\alpha)$ and $t_{0} \in \operatorname{supp}(\beta)$ such that

$$
\left(a_{s} b_{t} R\right) D^{-1} \subseteq\left(a_{s_{0}} b_{t_{0}} R\right) D^{-1} \quad \text { for any } s, t \in S .
$$

Set

$$
\begin{aligned}
& X=\left\{x \in \operatorname{supp}(\alpha): \exists u \in S \quad\left(a_{x} b_{u} R\right) D^{-1}=\left(a_{s_{0}} b_{t_{0}} R\right) D^{-1}\right\}, \\
& Y=\left\{y \in \operatorname{supp}(\beta): \exists v \in S \quad\left(a_{v} b_{y} R\right) D^{-1}=\left(a_{s_{0}} b_{t_{0}} R\right) D^{-1}\right\} .
\end{aligned}
$$

Since $S$ is a u.p. monoid and the sets $X$ and $Y$ are finite and nonempty (because $s_{0} \in X$ and $t_{0} \in Y$ ), there exist $x_{0} \in X$ and $y_{0} \in Y$ such that $x_{0} y_{0}$ is a u.p. element of $X Y$. Since $x_{0} \in X$ and $y_{0} \in Y$, for some $u_{0}, v_{0} \in S$ we have

$$
\left(a_{x_{0}} b_{u_{0}} R\right) D^{-1}=\left(a_{s_{0}} b_{t_{0}} R\right) D^{-1}
$$

and

$$
\left(a_{v_{0}} b_{y_{0}} R\right) D^{-1}=\left(a_{s_{0}} b_{t_{0}} R\right) D^{-1} .
$$


Assume that $\left(b_{u_{0}} R\right) D^{-1} \subseteq\left(b_{y_{0}} R\right) D^{-1}$. Then (4.2), Proposition 2.4 (e) and (4.1) imply that $\left(a_{s_{0}} b_{t_{0}} R\right) D^{-1}=\left(a_{x_{0}} b_{u_{0}} R\right) D^{-1} \subseteq\left(a_{x_{0}} b_{y_{0}} R\right) D^{-1} \subseteq\left(a_{s_{0}} b_{t_{0}} R\right) D^{-1}$, and thus

$$
\left(a_{x_{0}} b_{y_{0}} R\right) D^{-1}=\left(a_{s_{0}} b_{t_{0}} R\right) D^{-1}
$$

Since $\alpha \beta=0$, looking at the $x_{0} y_{0}$-coefficient of $\alpha \beta$ we obtain

$$
0=a_{x_{0}} b_{y_{0}}+c
$$

where $c$ is the sum of all products $a_{p} b_{q}$ with $p \in \operatorname{supp}(\alpha), q \in \operatorname{supp}(\beta), p q=x_{0} y_{0}$ and $(p, q) \neq\left(x_{0}, y_{0}\right)$ (if no such par $(p, q)$ exists, we set $\left.c=0\right)$. Note that for such a pair $(p, q)$, since $p q=x_{0} y_{0}$ and $x_{0} y_{0}$ is a u.p. element of $X Y$, and $(p, q) \neq\left(x_{0}, y_{0}\right)$, we have $p \notin X$ or $q \notin Y$. In either case, (4.1), (4.4), and the definitions of $X$ and $Y$ imply that $\left(a_{p} b_{q} R\right) D^{-1} \varsubsetneqq\left(a_{x_{0}} b_{y_{0}} R\right) D^{-1}$; so $\left(a_{p} b_{q} R\right) D^{-1} \subseteq\left(a_{x_{0}} b_{y_{0}} P\right) D^{-1}$ by Corollary $2.5(\mathrm{a})$, and thus

$$
a_{p} b_{q} \in\left(a_{x_{0}} b_{y_{0}} P\right) D^{-1}
$$

Since by Proposition 2.4 (b) the set $\left(a_{x_{0}} b_{y_{0}} P\right) D^{-1}$ is a right ideal of $R$, we have by (4.6) that $c \in\left(a_{x_{0}} b_{y_{0}} P\right) D^{-1}$, and thus (4.5) implies that $a_{x_{0}} b_{y_{0}} \in\left(a_{x_{0}} b_{y_{0}} P\right) D^{-1}$. Hence by Corollary 2.5 (b) we have $a_{x_{0}} b_{y_{0}} \in 0 D^{-1}$, and thus by (4.1) and (4.4), $a_{s} b_{t} \in 0 D^{-1}$ for all $s, t \in S$ in this case.

We are left with the case where $\left(b_{u_{0}} R\right) D^{-1} \nsubseteq\left(b_{y_{0}} R\right) D^{-1}$. Since the $D$-saturations of right ideals of $R$ are totally ordered, $\left(b_{y_{0}} R\right) D^{-1} \varsubsetneqq\left(b_{u_{0}} R\right) D^{-1}$ and we have that $\left(b_{y_{0}} R\right) D^{-1} \subseteq\left(b_{u_{0}} P\right) D^{-1}$ by Corollary $2.5(\mathrm{a})$. Hence Proposition $2.4(\mathrm{e})$ implies $\left(a_{v_{0}} b_{y_{0}} R\right) D^{-1} \subseteq\left(a_{v_{0}} b_{u_{0}} P\right) D^{-1}$, and from (4.1) and (4.3) we obtain

$$
a_{s} b_{t} \in\left(a_{v_{0}} b_{u_{0}} P\right) D^{-1} \quad \text { for any } s, t \in S \text {. }
$$

Applying (4.7) with $s=v_{0}$ and $t=u_{0}$, we obtain $a_{v_{0}} b_{u_{0}} \in\left(a_{v_{0}} b_{u_{0}} P\right) D^{-1}$, and by Corollary 2.5 (b) we have that $a_{v_{0}} b_{u_{0}} \in 0 D^{-1}$. Now another application of (4.7) yields $a_{s} b_{t} \in 0 D^{-1}$ for all $s, t \in S$, which completes the proof.

Proof of Theorem 4.1. Let $R$ be a right distributive ring, let $S$ be a u.p. monoid, and let $\alpha=\sum_{s \in S} a_{s} s, \beta=\sum_{t \in S} b_{t} t \in R[S]$ be such that $\alpha \beta=0$. Suppose that $a_{s} b_{t} \neq 0$ for some $s, t \in S$. Then $I=\left\{r \in R: a_{s} b_{t} r=0\right\}$ is a proper right ideal of $R$. Thus there exists a maximal right ideal $M$ of $R$ such that $I \subseteq M$. By Proposition 2.2, $M$ is a completely prime right ideal of $R$ and $D=R \backslash M$ is a right Ore subset of $R$, and by Theorem 3.1 the saturations of right ideals of $R$ with respect to $D$ are totally ordered. Hence by Lemma 4.2 there exists $d \in D$ with $a_{s} b_{t} d=0$. Consequently $d \in I$ and it follows that $d \in M$. This contradiction completes the proof in the case where $R$ is right distributive.

Assume now that $R$ is a left distributive ring and $S$ is a u.p. monoid. Then the opposite ring $R^{o p}$ of $R$ is right distributive and the opposite monoid $S^{o p}$ of $S$ is a u.p. monoid, and thus, as we already know, $R^{o p}$ is Armendariz relative to $S^{o p}$. Therefore, $R$ is Armendariz relative to $S$. 
By applying Theorem 4.1 to the additive monoid $S=\mathbb{N} \cup\{0\}$ of nonnegative integers, we obtain the following result.

Corollary 4.3. Every right or left distributive ring is Armendariz.

Recall that a ring $R$ is said to be a right chain ring if the right ideals of $R$ are totally ordered by set inclusion (see [4]). Left chain rings are defined similarly. Obviously, any right (resp. left) chain ring is right (resp. left) distributive and thus the following two results are immediate consequences of Theorem 4.1.

Corollary 4.4. (Corollary 6.2 in [15]) Every right or left chain ring is Armendariz relative to any u.p. monoid.

Corollary 4.5. (Corollary 6.3 in [15]) Every right or left chain ring is Armendariz.

For a ring $R$ and a polynomial $h \in R[x]$, let $c_{r}(h)$ denote the right ideal of $R$ generated by the coefficients of $h$. A ring $R$ is said to be right Gaussian if $c_{r}(f g)=c_{r}(f) c_{r}(g)$ for any $f, g \in R[x]$. The notion of right Gaussian rings, introduced in [17], extends to the noncommutative setting the notion of a commutative Gaussian ring. It is well known that every right Gaussian ring $R$ is right duo, that is, any right ideal of $R$ is a two-sided ideal (see Lemma 1.4 in [17]). Below we apply Theorem 4.1 to get a new and short proof of the following extension of the result (1.2) to noncommutative rings.

Corollary 4.6. (Theorem 2.1 in [17]) Every right duo right distributive ring is right Gaussian.

Proof. Let $R$ be a right duo right distributive ring. Obviously, all homomorphic images of $R$ are right distributive and thus, by Theorem 4.1, they are Armendariz. Now the result follows immediately from the known characterization of right Gaussian rings as those right duo rings whose homomorphic images are all Armendariz (see Theorem 1.5 in [17]).

\section{Armendariz rings relative to u.p. monoids are Armendariz, but not conversely}

In this section we show that for any nontrivial u.p. monoid $S$ the class of $S$ Armendariz rings is contained in the class of Armendariz rings, and we present an example of a u.p. monoid $S$ for which the containment is strict.

Proposition 5.1. If a ring $R$ is $S$-Armendariz for some nontrivial u.p. monoid $S$, then $R$ is Armendariz.

Proof. Since $S$ is nontrivial, we can choose $t \in S \backslash\{1\}$. Let $T$ be the submonoid of $S$ generated by $t$, i.e., $T=\left\{1, t, t^{2}, \ldots\right\}$. Since $R$ is $S$-Armendariz, $R$ is $T$-Armendariz as well, and thus to show that $R$ is Armendariz, it suffices to show that the 
monoid $T$ is isomorphic to the additive monoid $\mathbb{N} \cup\{0\}$. In turn, to show this, it is enough to prove that for any $i, j \in \mathbb{N} \cup\{0\}, i \neq j$ implies $t^{i} \neq t^{j}$. Suppose that $i \neq j$ but $t^{i}=t^{j}$, where without loss of generality we can assume that $i>j$. Then, since each u.p. monoid is cancellative, we have $t^{n}=1$ for $n=i-j \in \mathbb{N}$ (note that $n \geq 2$, since $t \neq 1)$. Now for the subsets $X=\{1, t\}$ and $Y=\left\{1, t, t^{2}, \ldots, t^{n-1}\right\}$ of $S$ we can easily see that there does not exist an element of $S$ written uniquely in the form $x y$ with $x \in X$ and $y \in Y$, contradicting that $S$ is a u.p. monoid.

By Proposition 5.1, all Armendariz rings relative to a nontrivial u.p. monoid are Armendariz. In the example below we show that, in general, the converse is not true.

Example 5.2. There exist a u.p. monoid $S$ and a ring $R$ such that $R$ is Armendariz but not $S$-Armendariz.

Let $S$ be the monoid generated by $s_{1}, s_{2}, s_{3}, t_{1}, t_{2}, t_{3}$ with the following defining relations:

$$
s_{1} t_{1}=s_{2} t_{3}, \quad s_{1} t_{2}=s_{3} t_{1}, \quad s_{1} t_{3}=s_{2} t_{2}, \quad s_{3} t_{2}=s_{2} t_{1} .
$$

As it was shown by J. Krempa, $S$ is a u.p. monoid (see Example 13 of Chapter 10 in $[18])$.

Let $K=\mathbb{Z}_{2}$ be the field of two elements and let $R$ be the $K$-algebra generated by $y_{1}, y_{2}, y_{3}$ with the following defining relations:

$$
\begin{aligned}
& y_{1}^{2}=y_{2} y_{3}, \quad y_{1} y_{2}=y_{3} y_{1}, \quad y_{1} y_{3}=y_{2}^{2}, \quad y_{3} y_{2}=y_{2} y_{1}, \\
& y_{3}^{2}=0, \quad y_{i} y_{j} y_{k}=0 \quad \text { for all } i, j, k \in\{1,2,3\} .
\end{aligned}
$$

We will show that the ring $R$ is Armendariz but not $S$-Armendariz. We will show even more, namely that the ring $R$ is power-serieswise Armendariz (in the sense of [11]), i.e., for any power series $f=\sum_{i=0}^{\infty} a_{i} x^{i}, g=\sum_{j=0}^{\infty} b_{j} x^{j} \in R[[x]]$, if $f g=0$, then $a_{i} b_{j}=0$ for all $i$ and $j$. Obviously, any power-serieswise Armendariz ring is Armendariz.

Notice that by the defining relations (5.1), any product $y_{i} y_{j}$ with $(i, j) \neq(3,3)$ can be represented as one of $y_{2} y_{3}, y_{3} y_{1}, y_{2}^{2}$, or $y_{2} y_{1}$ (we use the right sides of the defining relations (5.1) as representatives for the products). This and the diamond lemma (see [3]) imply that every element $a \in R$ can be uniquely written as a linear combination over $K$ of elements of the set $B=\left\{1, y_{1}, y_{2}, y_{3}, y_{2} y_{3}, y_{3} y_{1}, y_{2}^{2}, y_{2} y_{1}\right\}$. Hence the ring $R$ is a $\mathbb{Z}_{2}$-algebra of dimension 8 , so $R$ has the additional feature of being finite (of order $2^{8}$ ). The coefficient from $K$ with which $1 \in B$ appears when $a \in R$ is written as a linear combination of elements of $B$ will be called the constant term of $a$. It is clear that the set $J$ of elements of $R$ whose constant term is equal to zero, is an ideal of $R$,

$$
J^{2}=\left\{k_{1} y_{2} y_{3}+k_{2} y_{3} y_{1}+k_{3} y_{2}^{2}+k_{4} y_{2} y_{1}: k_{1}, k_{2}, k_{3}, k_{4} \in K\right\}
$$

and $J^{3}=\{0\}$. Hence if $a \in R \backslash J$ (i.e., the constant term of $a \in R$ is nonzero), then $a=1+j$ for some $j \in J$ and thus $a$ is invertible in $R$. Therefore, $R$ is a local ring and $J$ is the unique maximal right ideal of $R$. 
To show that the $\operatorname{ring} R$ is not $S$-Armendariz, we consider the elements $\alpha=$ $y_{1} s_{1}+y_{2} s_{2}+y_{3} s_{3}$ and $\beta=y_{1} t_{1}+y_{2} t_{2}+y_{3} t_{3}$ of the monoid ring $R[S]$. Then $\alpha \beta=0$, but the product $y_{1} y_{1}$ of the $s_{1}$-coefficient of $\alpha$ and the $t_{1}$-coefficient of $\beta$ is nonzero. Thus $R$ is not $S$-Armendariz.

Now we show that the ring $R$ is power-serieswise Armendariz. Let

$$
f=\sum_{i=0}^{\infty} a_{i} x^{i} \quad \text { and } \quad g=\sum_{j=0}^{\infty} b_{j} x^{j} \in R[[x]]
$$

be such that $f g=0$, i.e.,

$$
\left(a_{0}+a_{1} x+a_{2} x^{2}+\cdots\right)\left(b_{0}+b_{1} x+b_{2} x^{2}+\cdots\right)=0 .
$$

To show that $a_{i} b_{j}=0$ for all $i$ and $j$, we consider three cases.

Case 1: $a_{k} \notin J$ or $b_{k} \notin J$ for some $k \in \mathbb{N} \cup\{0\}$. Assume that $a_{k} \notin J$ for some $k$, and let $k$ be minimal with this property. Then $a_{k}$ is an invertible element of $R$. Furthermore, $a_{i} \in J$ for any $i<k$, and thus by considering the equation (5.3) modulo the ideal

$$
J[[x]]=\left\{\sum_{m=0}^{\infty} c_{m} x^{m} \in R[[x]]: \forall_{m} c_{m} \in J\right\}
$$

of $R[[x]]$, we obtain

$$
\left(a_{k} x^{k}+a_{k+1} x^{k+1}+a_{k+2} x^{k+2}+\cdots\right)\left(b_{0}+b_{1} x+b_{2} x^{2}+\cdots\right) \equiv 0(\bmod J[[x]])
$$

and after canceling both sides of (5.4) by $x^{k}$, we get

$$
\left(a_{k}+a_{k+1} x+a_{k+2} x^{2}+\cdots\right)\left(b_{0}+b_{1} x+b_{2} x^{2}+\cdots\right) \equiv 0(\bmod J[[x]]) .
$$

Since $a_{k}$ is invertible in $R$, it is invertible modulo $J$, so the power series $a_{k}+a_{k+1} x+$ $a_{k+2} x^{2}+\cdots$ is invertible modulo $J[[x]]$ in $R[[x]]$. Now it follows from (5.5) that $b_{0}+b_{1} x+b_{2} x^{2}+\cdots \equiv 0(\bmod J[[x]])$, and so $b_{j} \in J$ for any $j$. Hence $a_{i} b_{j} \in J^{2}$ for any $i<k$ and any $j$, and thus

$$
\left(a_{0}+a_{1} x+a_{2} x^{2}+\cdots+a_{k-1} x^{k-1}\right)\left(b_{0}+b_{1} x+b_{2} x^{2}+\cdots\right) \equiv 0\left(\bmod J^{2}[[x]]\right) .
$$

Therefore, (5.3) implies that

$$
\left(a_{k}+a_{k+1} x+a_{k+2} x^{2}+\cdots\right)\left(b_{0}+b_{1} x+b_{2} x^{2}+\cdots\right) \equiv 0\left(\bmod J^{2}[[x]]\right) .
$$

Since $a_{k}$ is invertible in $R$, it is invertible modulo $J^{2}$ as well, so it follows that $b_{0}+b_{1} x+b_{2} x^{2}+\cdots \equiv 0\left(\bmod J^{2}[x]\right)$, and thus $b_{j} \in J^{2}$ for any $j$. Since $J^{3}=\{0\}$, it follows that

$$
\left(a_{0}+a_{1} x+a_{2} x^{2}+\cdots+a_{k-1} x^{k-1}\right)\left(b_{0}+b_{1} x+b_{2} x^{2}+\cdots\right)=0,
$$

so from (5.3) we obtain

$$
\left(a_{k}+a_{k+1} x+a_{k+2} x^{2}+\cdots\right)\left(b_{0}+b_{1} x+b_{2} x^{2}+\cdots\right)=0 .
$$

Since $a_{k}$ is invertible in $R$, the power series $a_{k}+a_{k+1} x+a_{k+2} x^{2}+\cdots$ is invertible in $R[[x]]$, and it follows that $b_{j}=0$ for any $j$. Thus $a_{i} b_{j}=0$ for any $i$ and $j$.

The subcase of Case 1 where $b_{k} \notin J$ for some $k$ can be treated similarly. 
If the Case 1 does not occur, then $a_{i} \in J$ for any $i$ and $b_{j} \in J$ for any $j$. Hence in the remaining we assume that $a_{i}, b_{j} \in J$ for all $i$ and $j$.

In other words, we assume that for any $i$ and $j$ the constant terms of $a_{i}$ and $b_{j}$ are equal to zero.

Case 2: Either $a_{i} \in J^{2}$ for any $i \in \mathbb{N} \cup\{0\}$, or $b_{j} \in J^{2}$ for any $j \in \mathbb{N} \cup\{0\}$. In this case for any $i$ and $j$ we have $a_{i} b_{j} \in J^{3}$, so $a_{i} b_{j}=0$.

If neither Case 1 nor Case 2 occurs, then we have the following situation.

Case 3: There exist $m, n \in \mathbb{N} \cup\{0\}$ such that $a_{m}, b_{n} \in J \backslash J^{2}$. Let $m$ be minimal with $a_{m} \in J \backslash J^{2}$, and let $n$ be minimal with $b_{n} \in J \backslash J^{2}$. Then $a_{i} \in J^{2}$ for any $i<m$, and $b_{j} \in J^{2}$ for any $j<n$, and since $J^{3}=\{0\}$, the equation (5.3) reduces to $\left(a_{m} x^{m}+a_{m+1} x^{m+1}+a_{m+2} x^{m+2}+\cdots\right)\left(b_{n} x^{n}+b_{n+1} x^{n+1}+b_{n+2} x^{n+2}+\cdots\right)=0$.

After cancelling both sides of the above equation by $x^{m+n}$, we obtain

$$
\left(a_{m}+a_{m+1} x+a_{m+2} x^{2}+\cdots\right)\left(b_{n}+b_{n+1} x+b_{n+2} x^{2}+\cdots\right)=0 .
$$

For any $i, j \in \mathbb{N} \cup\{0\}$, if $i<m$ or $j<n$, then we have $a_{i} b_{j} \in J^{3}=\{0\}$, and thus to prove that $a_{i} b_{j}=0$ for all $i$ and $j$, we can assume that $m=n=0$. Therefore, we assume the equality (5.3) with $a_{0}, b_{0} \in J \backslash J^{2}$ and $a_{i}, b_{j} \in J$ for all $i$ and $j$, and we have to show that $a_{i} b_{j}=0$ for any $i$ and $j$.

Since $a_{0}, b_{0} \in J \backslash J^{2}$, there exist triples $\left(k_{1}, k_{2}, k_{3}\right),\left(l_{1}, l_{2}, l_{3}\right) \in K^{3} \backslash\{(0,0,0)\}$ and $p, q \in J^{2}$ such that $a_{0}=k_{1} y_{1}+k_{2} y_{2}+k_{3} y_{3}+p$ and $b_{0}=l_{1} y_{1}+l_{2} y_{2}+l_{3} y_{3}+q$. Since $f g=0$, we have

$$
\begin{aligned}
0 & =a_{0} b_{0}=\left(k_{1} y_{1}+k_{2} y_{2}+k_{3} y_{3}+p\right)\left(l_{1} y_{1}+l_{2} y_{2}+l_{3} y_{3}+q\right) \\
& =\left(k_{1} l_{1}+k_{2} l_{3}\right) y_{2} y_{3}+\left(k_{1} l_{2}+k_{3} l_{1}\right) y_{3} y_{1}+\left(k_{1} l_{3}+k_{2} l_{2}\right) y_{2}^{2}+\left(k_{2} l_{1}+k_{3} l_{2}\right) y_{2} y_{1} .
\end{aligned}
$$

Hence in the equation above, all the "coefficients" of $y_{2} y_{3}, y_{3} y_{1}, y_{2}^{2}$, and $y_{2} y_{1}$ are equal to zero, and since $R$ is a $K$-algebra of characteristic 2 , we obtain the equations:

$$
\begin{aligned}
& k_{1} l_{1}=k_{2} l_{3}, \\
& k_{1} l_{2}=k_{3} l_{1}, \\
& k_{1} l_{3}=k_{2} l_{2}, \\
& k_{2} l_{1}=k_{3} l_{2} .
\end{aligned}
$$

We consider two subcases.

Subcase A: $k_{1} \neq 0$ and $k_{2} \neq 0$. Then $k_{1}=k_{2}=1$, so (5.6) and (5.8) imply $l_{1}=l_{2}=l_{3}$, and since $b_{0} \notin J^{2}$, it follows that $l_{1}=l_{2}=l_{3}=1$. Hence $k_{3}=1$ by (5.7). Therefore, $a_{0}=y_{1}+y_{2}+y_{3}+p$ and $b_{0}=y_{1}+y_{2}+y_{3}+q$ for some $p, q \in J^{2}$.

Next we concentrate on the coefficients $a_{1}$ and $b_{1}$. Since $a_{1}, b_{1} \in J$, we have

$$
a_{1}=u_{1} y_{1}+u_{2} y_{2}+u_{3} y_{3}+s \quad \text { for some } u_{1}, u_{2}, u_{3} \in K \text { and } s \in J^{2},
$$


and

$$
b_{1}=v_{1} y_{1}+v_{2} y_{2}+v_{3} y_{3}+t \quad \text { for some } v_{1}, v_{2}, v_{3} \in K \text { and } t \in J^{2} .
$$

Since $f g=0$, from the $x$-coefficient of the product $f g$, we obtain

$$
a_{0} b_{1}+a_{1} b_{0}=0
$$

and thus

$$
\begin{aligned}
0= & a_{0} b_{1}+a_{1} b_{0} \\
= & \left(y_{1}+y_{2}+y_{3}+p\right)\left(v_{1} y_{1}+v_{2} y_{2}+v_{3} y_{3}+t\right) \\
& +\left(u_{1} y_{1}+u_{2} y_{2}+u_{3} y_{3}+s\right)\left(y_{1}+y_{2}+y_{3}+q\right) \\
= & \left(v_{1}+v_{3}+u_{1}+u_{2}\right) y_{2} y_{3}+\left(v_{2}+v_{1}+u_{1}+u_{3}\right) y_{3} y_{1} \\
& +\left(v_{3}+v_{2}+u_{1}+u_{2}\right) y_{2}^{2}+\left(v_{1}+v_{2}+u_{2}+u_{3}\right) y_{2} y_{1} .
\end{aligned}
$$

Hence

$$
\begin{aligned}
& v_{1}+v_{3}=u_{1}+u_{2}, \\
& v_{2}+v_{1}=u_{1}+u_{3}, \\
& v_{3}+v_{2}=u_{1}+u_{2}, \\
& v_{1}+v_{2}=u_{2}+u_{3} .
\end{aligned}
$$

From (5.13) and (5.15) it follows that $v_{1}=v_{2}$, and thus $v_{1}+v_{2}=0$. Now we deduce from (5.14) and (5.16) that $u_{1}=u_{2}=u_{3}$. Hence (5.13) implies $v_{1}=v_{2}=v_{3}$. Therefore, $a_{1}, b_{1} \in K\left(y_{1}+y_{2}+y_{3}\right)+J^{2}$. Note that $\left(y_{1}+y_{2}+y_{3}\right)^{2}=0$, hence

$$
\left[K\left(y_{1}+y_{2}+y_{3}\right)+J^{2}\right] \cdot\left[K\left(y_{1}+y_{2}+y_{3}\right)+J^{2}\right]=\{0\},
$$

and thus $a_{1} b_{1}=0$.

To get information on the coefficients $a_{2}$ and $b_{2}$, we look at the $x^{2}$-coefficient of the product $f g=0$, obtaining that $a_{0} b_{2}+a_{1} b_{1}+a_{2} b_{0}=0$. Since $a_{1} b_{1}=0$, it follows that $a_{0} b_{2}+a_{2} b_{0}=0$, and we are in the circumstances of the equation (5.12), with $a_{1}$ replaced by $a_{2}$, and $b_{1}$ replaced by $b_{2}$. Hence, by the same argument as above, we obtain that

$$
a_{2}, b_{2} \in K\left(y_{1}+y_{2}+y_{3}\right)+J^{2},
$$

and thus it follows from (5.17) that $a_{1} b_{2}=a_{2} b_{1}=0$.

Next we look at the $x^{3}$-coefficient of the product $f g=0$, obtaining

$$
0=a_{0} b_{3}+a_{1} b_{2}+a_{2} b_{1}+a_{3} b_{0}=a_{0} b_{3}+a_{3} b_{0},
$$

which implies that $a_{3}, b_{3} \in K\left(y_{1}+y_{2}+y_{3}\right)+J^{2}$.

Continuing this way we obtain for any $i$ and $j$ that $a_{i}, b_{j} \in K\left(y_{1}+y_{2}+y_{3}\right)+J^{2}$, and thus $a_{i} b_{j}=0$ by $(5.17)$. 
Subcase B: $k_{1}=0$ or $k_{2}=0$. If $k_{1} \neq 0$, then $l_{1}=0$ by (5.6) and $l_{3}=0$ by (5.8), so also $l_{2}=0$ by (5.7). This contradiction shows that $k_{1}=0$, and similarly one can show that $k_{2}=0$. Since $k_{1}=k_{2}=0$ and $a_{0} \in J \backslash J^{2}$, it follows that $k_{3}=1$, and thus (5.7) and (5.9) imply $l_{1}=l_{2}=0$. Hence $l_{3}=1$, and thus $a_{0}=y_{3}+p$ and $b_{0}=y_{3}+q$ for some $p, q \in J^{2}$.

Next we look at the coefficients $a_{1}$ and $b_{1}$, which as in Subcase A can be written in the forms (5.10) and (5.11), respectively. Now (5.12) gives

$$
\begin{aligned}
0 & =\left(y_{3}+p\right)\left(v_{1} y_{1}+v_{2} y_{2}+v_{3} y_{3}+t\right)+\left(u_{1} y_{1}+u_{2} y_{2}+u_{3} y_{3}+s\right)\left(y_{3}+q\right)= \\
& =v_{1} y_{3} y_{1}+v_{2} y_{2} y_{1}+u_{1} y_{2}^{2}+u_{2} y_{2} y_{3}
\end{aligned}
$$

and $v_{1}=v_{2}=u_{1}=u_{2}=0$ follows. Hence $a_{1}, b_{1} \in K y_{3}+J^{2}$, and since

$$
\left(K y_{3}+J^{2}\right) \cdot\left(K y_{3}+J^{2}\right)=\{0\},
$$

we obtain $a_{1} b_{1}=0$. Following the same pattern as in Subcase A, we obtain that $a_{i}, b_{j} \in K y_{3}+J^{2}$ for any $i, j$, and thus $a_{i} b_{j}=0$ by (5.18).

\section{References}

[1] Anderson, D. D. And Camillo, V.: Armendariz rings and Gaussian rings. Comm. Algebra 26 (1998), 2265-2272.

[2] Armendariz, E. P.: A note on extensions of Baer and P.P.-rings. J. Austral. Math. Soc. 18 (1974), 470-473.

[3] Bergman, G. M.: The diamond lemma for ring theory. Adv. in Math. 29 (1978), no. $2,178-218$

[4] Bessenrodt, C., Brungs, H. H. and Törner, G.: Right chain rings, Part 1. Schriftenreihe des Fachbereichs Math. 181, Unversität Duisburg, 1990.

[5] Birkenmeier, G. F. And Park, J. K.: Triangular matrix representations of ring extensions. J. Algebra 265 (2003), 457-477.

[6] FAith, C.: Rings and things and a fine array of twentieth century associative algebra. Mathematical Surveys and Monographs 65, American Mathematical Society, Providence, RI, 1999.

[7] Ferrero, M. and Sant'Ana, A.: On distributive modules and rings. Results Math. 44 (2003), 74-85.

[8] Fuchs, L.: Über die Ideale arithmetischer Ringe. Comment. Math. Helv. 23 (1949), $334-341$.

[9] Glaz, S. And Schwarz, R.: Prüfer conditions in commutative rings. Arab. J. Sci. Eng. 36 (2011), 967-983.

[10] Jensen, C. U.: A remark on arithmetical rings. Proc. Amer. Math. Soc. 15 (1964), 951-954.

[11] Kim, N.K., Lee, K.H. And Lee, Y.: Power series rings satisfying a zero divisor property. Comm. Algebra 34 (2006), 2205-2218.

[12] LAM, T. Y.: Lectures on modules and rings. Graduate Texts in Mathematics 189, Springer-Verlag, New York, 1999. 
[13] Liu, Z.: Armendariz rings relative to a monoid. Comm. Algebra 33 (2005), 649-661.

[14] Marks, G., Mazurek, R. And Ziembowski, M.: A new class of unique product monoids with applications to ring theory. Semigroup Forum 78 (2009), 210-225.

[15] Marks, G., Mazurek, R. And Ziembowski, M.: A unified approach to various generalizations of Armendariz rings. Bull. Aust. Math. Soc. 81 (2010), 361-397.

[16] Mazurek, R. And PuczyŁowski, E. R.: On nilpotent elements of distributive rings. Comm. Algebra 18 (1990), 463-471.

[17] Mazurek, R. And Ziembowski, M.: Right Gaussian rings and skew power series rings. J. Algebra 330 (2011), 130-146.

[18] Okninski, J.: Semigroup algebras. Monographs and Textbooks in Pure and Applied Mathematics 138, Marcel Dekker, New York, 1991.

[19] Puninski, G.: Projective modules over the endomorphism ring of a biuniform module. J. Pure Appl. Algebra 188 (2004), 227-246.

[20] Rege, M. B. and Chhawchharia, S.: Armendariz rings. Proc. Japan Acad. Ser. A Math. Sci. 73 (1997), 14-17.

[21] Stephenson, W.: Modules whose lattice of submodules is distributive. Proc. Lond. Math. Soc. (3) 28 (1974), 291-310.

[22] Törner, G. And Zima, J.: Some remarks on linear structures in right distributive domains. Forum Math. 11 (1999), 1-15.

[23] Tuganbaev, A. A.: Semidistributive modules and rings. Mathematics and its Applications 449, Kluwer Academic Publishers, Dordrecht, 1998.

Received November 7, 2012.

Ryszard Mazurek: Faculty of Computer Science, Bialystok University of Technology, Wiejska 45A, 15-351 Białystok, Poland.

E-mail: r.mazurek@pb.edu.pl

Michą Ziembowski: Faculty of Mathematics and Information Science, Warsaw University of Technology, Koszykowa 75, 00-662 Warsaw, Poland.

E-mail: m.ziembowski@mini.pw.edu.pl

The research of the first author was supported by the Polish National Center of Science Grant no. DEC-2011/03/B/ST1/04893. The second author was supported by the Foundation for Polish Science-Homing Plus Programme, Homing Plus BIS/2011-3/2. 